BMJ Open Diabetes

Research \& Care

\title{
Adherence to antidiabetic treatment and impaired hypoglycemia awareness in type 2 diabetes mellitus assessed in Spanish community pharmacies: the ADHIFAC study
}

\author{
Fernando Gomez-Peralta (D) , ${ }^{1}$ José A Fornos Pérez, ${ }^{2,3}$ Ana Molinero, ${ }^{3,4}$ \\ Ignacio M Sánchez Barrancos, ${ }^{5}$ Ezequiel Arranz Martínez, ${ }^{6}$ Pablo Martínez-Pérez, ${ }^{7}$ \\ Inés Mera Gallego, ${ }^{3,8}$ N Floro Andrés-Rodríguez ${ }^{3,9}$
}

To cite: Gomez-Peralta F, Fornos Pérez JA, Molinero A, et al. Adherence to antidiabetic treatment and impaired hypoglycemia awareness in type 2 diabetes mellitus assessed in Spanish community pharmacies: the ADHIFAC study. BMJ Open Diab Res Care 2021;9:e002148. doi:10.1136/ bmjdrc-2021-002148

- Additional supplemental material is published online only. To view, please visit the journal online (http://dx.doi. org/10.1136/bmjdrc-2021002148)

Received 9 July 2021 Accepted 19 0ctober 2021

Check for updates

C Author(s) (or their employer(s)) 2021. Re-use permitted under CC BY-NC. No commercial re-use. See rights and permissions. Published by BMJ.

For numbered affiliations see end of article.

Correspondence to Dr Fernando Gomez-Peralta; fgomezperalta@gmail.com

\section{ABSTRACT}

Introduction Adherence to treatment and hypoglycemia awareness are strongly linked to glycemic control and hypoglycemia risk in people with type 2 diabetes mellitus (T2DM). Community pharmacies are suitable facilities to detect these conditions, and should be involved in the strategies to minimize the associated risks and burden.

Research design and methods This cross-sectional study conducted at community pharmacies across Spain assessed the prevalence of low adherence to antidiabetic treatments, the frequency of impaired hypoglycemia awareness, and their predictive factors. Adherence was measured with the 8-item Morisky Medication Adherence Scale (MMAS-8) and electronic records of dispensed treatments. The Clarke questionnaire was used to assess impaired hypoglycemia awareness. Healthcare counseling provided in the pharmacy was collected.

Results Seventy-nine pharmacists and 618 subjects with T2DM participated in the study. Mean age in the overall T2DM population was 67 years, being the majority (69\%) pensioners. Adherence was high in $41 \%$ of participants, medium in $35 \%$, and low in $24 \%$ according to the MMAS-8. Impaired hypoglycemia awareness was observed in $25 \%$ of participants. Main determinants of low adherence were the level of education, the number of treatments per patient, hypoglycemia awareness, and the type of pharmacy. Predictive factors of impaired hypoglycemia awareness were the level of education, information on diabetes-related complications, adherence levels, and the type of pharmacy. The proportion of participants who had healthcare counseling was $71 \%$ in the overall population and $100 \%$ in subjects with impaired hypoglycemia awareness and low adherence. Healthcare counseling comprised diabetes education (69\%), pharmacotherapeutic assessment (20\%), and physician referrals (11\%).

Conclusion Lack of adherence to antidiabetic treatments and impaired hypoglycemia awareness are frequent and correlate in T2DM. Community pharmacies can detect these conditions and should have an active role in the design of strategies to minimize them.

\section{Significance of this study}

What is already known about this subject?

- Low adherence and impaired hypoglycemia awareness are the main barriers to achieve good metabolic control and prevent long-term complications in people with type 2 diabetes mellitus (T2DM).

- Community pharmacists are key professionals to help manage T2DM because of their high accessibility and vast expertise on dose regimens and treatment-related adverse events.

- Community pharmacies can be crucial facilities to promote initiatives aiming to increase adherence to antidiabetic treatments and hypoglycemia awareness.

What are the new findings?

- This large cross-sectional study confirmed the low antidiabetic treatment adherence and significant prevalence $(25 \%)$ of impaired hypoglycemia awareness in T2DM people attended at community pharmacies.

- Main determinants of low adherence were a lower education level, the number of treatments per patient, hypoglycemia unawareness, and the type of pharmacy.

- Predictive factors of impaired hypoglycemia awareness were a lower education level, the presence of other diabetes-related complications, low adherence levels, and the type of pharmacy.

How might these results change the focus of research or clinical practice?

- Further efforts should be made to mitigate low adherence and impaired hypoglycemia awareness, considering predictive factors identified in the T2DM population.

- As adherence and hypoglycemia awareness were effectively evaluated in community pharmacies, actions to improve these conditions may be conducted at these facilities. 


\section{INTRODUCTION}

Diabetes mellitus is a complex chronic disease and a major public health problem, imposing a substantial burden on patients and healthcare systems. ${ }^{1}$ The prevalence of diabetes mellitus worldwide was 451 million people in 2017 and is expected to increase to 693 million by $2045 .^{2}$ The most common form is type 2 diabetes mellitus (T2DM), accounting for $90 \%-95 \%$ of cases. ${ }^{3}$ In Spain, it was estimated that $13.8 \%$ of people have T2DM, ${ }^{4}$ with a high proportion $(51.4 \%)$ poorly controlling the disease. $^{5}$

T2DM requires complex pharmacological interventions to manage hyperglycemia and diabetes-associated complications. ${ }^{6}$ Because of treatment complexity and the required lifetime self-management, adherence continues to be a challenge and a leading barrier to treatment success. Significant efforts have been made to identify factors associated with non-adherence and implement interventions to prevent its consequences. ${ }^{7}$ The main determinants of low adherence to antidiabetic treatments found in previous studies are socioeconomic factors, the complexity of treatments, and fear of hypoglycemia. ${ }^{89}$

Hypoglycemia, and fear of hypoglycemia, are yet other important barriers to achieve good metabolic control, ${ }^{10}$ representing a considerable human and economic burden. ${ }^{11}$ Avoiding hypoglycemia can prevent treatment discontinuations and quality-of-life impairment and is, therefore, one of the main goals of treatment interventions. ${ }^{12}$ As disease advances, recurrent hypoglycemic episodes may lead to a progressive impairment in the ability to recognize symptoms associated with low blood glucose. ${ }^{13}$ Unrecognized hypoglycemic events can ultimately result in severe hypoglycemia, ${ }^{14}$ a clinical challenge with devastating consequences (including seizures, loss of consciousness, and hospital admissions) ${ }^{15}$ Therefore, strategies to promote the recognition and interpretation of symptoms associated with hypoglycemia are highly advisable.

Given the complex and multifactorial nature of T2DM, involving multidisciplinary teams in patient care is crucial. Among this team, community pharmacists are key professionals with high accessibility, close contact with the patient, and vast expertise on dose regimens and treatment-related adverse events. Community pharmacies are natural locations for prevention, education, and disease monitoring that can improve adherence to antidiabetic treatments, reduce unrecognized hypoglycemic episodes, and minimize treatment-related adverse events. $^{16}$

To implement these strategies, it is necessary to gain knowledge on the prevalence of non-adherence and impaired awareness of hypoglycemia (IAH) and identify their predictive factors. However, no previous study evaluated these aspects at community pharmacies across Spain. For this reason, the Spanish Society of Clinical, Family and Community Pharmacy (SEFAC) ${ }^{17}$ the Spanish Diabetes Society (SED) ${ }^{18}$ the Spanish Society of Primary Care Physicians (SEMERGEN), ${ }^{19}$ and the Spanish Society of Family and Community Medicine (semFYC) $)^{20}$ proposed to investigate the prevalence of low adherence to antidiabetic treatments and the frequency of unrecognized hypoglycemic events in people with T2DM attended at community pharmacies.

\section{METHODS}

\section{Study design}

This observational, cross-sectional, multicenter study was conducted at 79 community pharmacies in Spain. The study adhered to the tenets of the Declaration of Helsinki. After classification by the Spanish Agency for Medicines and Health Products (AEMPS), the study was approved by the Clinical Research Ethics Committee of Fundació Sant Joan de Déu. All participants provided written informed consent before starting any assessment associated with the study.

The protocol of the study has been previously published. ${ }^{21}$ In brief, data were obtained from interviews conducted at community pharmacies across the country. Information collected included demographic and clinical characteristics of study participants, data on previous follow-ups, and comorbidities. To evaluate treatment adherence to antidiabetic treatments, participants completed the Spanish validated version of the 8-item Morisky Medication Adherence Scale (MMAS-8) ${ }^{22-25}$ and the electronic records of dispensed treatments were reviewed. The Spanish validated version of the Clarke test was also administered to participants treated with hypoglycemic antidiabetic treatments (sulfonylureas, glinides, and insulin) to evaluate the level of hypoglycemia awareness. ${ }^{26}$ Pharmacists who agreed to participate received a 1-day training (on-site or online) session by the Scientific Steering Committee. The training covered the rationale of the study, protocol details, and instructions on how to administer the questionnaires to standardize the implementation of study procedures and avoid bias.

\section{Study population}

The investigators consecutively recruited adult participants receiving pharmacologic antidiabetic treatment, following the same therapeutic regimen for at least 12 months, with an e-prescription in the previous 6 months and who agreed to participate in the study. ${ }^{21}$ Hypoglycemia awareness was evaluated in eligible participants treated with sulfonylureas, glinides or insulin.

Participants who were unable to understand the procedures of the study or complete the questionnaires were excluded from the study. Participating pharmacists were members of the SEFAC professional association.

\section{Study outcomes}

The aim of this study was to evaluate the feasibility of measuring and initiating actions for improving T2DM treatment adherence in community pharmacies. Secondary objectives were to assess the frequency of IAH 
and to identify predictive factors of low adherence to antidiabetic treatments and IAH.

The MMAS- 8 is a modified version with eight items of the MMAS, ${ }^{27}$ a self-reported measure of adherence (online supplemental figure 1). ${ }^{23}$ The respondent is required to answer "yes" or "no" to seven items and to rate the last item on a 5-point Likert scale. Scores can range between 0 and 8 and are classified into low (score $<6$ ), medium (score 6-7), and high adherence (score of 8). The Spanish validated version of the MMAS- 8 was used for this study. ${ }^{22}$

Adherence was also calculated based on electronic records of medications dispensed at community pharmacies. High adherence was established when $100 \%$ of the prescribed medication was collected within the last 6 months, medium if $75 \%-99 \%$ of the prescribed medication was collected, and low when $<75 \%$ of the prescribed medication was collected.

The Clarke test evaluates the frequency and severity of hypoglycemic events. The questionnaire comprises eight questions characterizing the frequency of hypoglycemic events, hypoglycemic symptoms, and the correlation with blood glucose levels. Responses are designated as $\mathrm{R}$ or A. A sum of $\mathrm{R}$ responses $\geq 4$ indicates $\mathrm{IAH}, \mathrm{R}=3$ undetermined awareness, and $\mathrm{R} \leq 2$ adequate hypoglycemia awareness (online supplemental figure 2) ${ }^{13}$ Participants were categorized into those with IAH (Clarke test $\geq 4$ ) or non-IAH (Clarke test $\leq 3$ ).

The investigators recorded the healthcare counseling initiated at community pharmacies when low adherence or IAH were detected, and results were reported for patients with impaired hypoglycemia awareness and low adherence. Diabetes education comprised information leaflets on diabetes and hypoglycemia management for adherent and hypoglycemia aware participants, and more specific information on the importance of adhering to prescribed treatments for non-adherent participants. Information on how to detect and manage hypoglycemia was provided to participants with IAH. Following the protocol, ${ }^{21}$ participants with low adherence and/or with IAH were referred to their specialist for appropriate care via a physician referral form. Pharmacotherapeutic counseling comprised information on the correct use of T2DM treatment and posology.

\section{Statistical analyses}

Analyses were performed with the software STATA MP13.0. Logistic regression models were used to assess predictors of non-adherence to antidiabetic agents and of IAH. We first performed simple regression models and those variables reaching statistical significance and meaningful effect size were included in the multivariable logistic regression model. The level of significance set for variable selection was $\mathrm{p}<0.20$ and the meaningful effect size as OR $>1.1$ or $<0.9$ for categorical variables and OR $>1.01$ or $<0.99$ for continuous variables. In the multivariable model, a $\mathrm{p}$ value $<0.05$ was considered significant. Multicollinearity was tested using a variance inflation factor. Those variables with a variance inflation factor $>5$ were removed from the models.

Differences between women and men were analyzed with the $\chi^{2}$ test for categorical variables and Student's t-test for continuous variables. The level of statistical significance was set at $\mathrm{p}<0.05$.

The sample size was calculated to achieve a 5.0\% accuracy with $95 \%$ bilateral correction for finite populations considering an average $50 \%$ adherence reported in previous studies. ${ }^{28}{ }^{29}$ Considering a $50 \%$ adherence and a target population of 5158754 adults with diabetes mellitus, ${ }^{4}$ of whom 3611128 would be treated pharmacologically, and a dropout rate $<20 \%, 462$ participants were required from at least 50 pharmacies. ${ }^{21}$

A large cohort study ${ }^{30}$ showed a frequency of hypoglycemic episodes of $5 \%$. Considering this prevalence, 319 participants were required for the analysis of hypoglycemia awareness. ${ }^{21}$

\section{RESULTS}

\section{Investigator pharmacists}

Seventy-nine pharmacists participated in the study; $77.2 \%$ worked in an urban pharmacy and $22.8 \%$ in a rural pharmacy. Mean working experience was 16.7 years, most were women $(65.8 \%)$, and mean age was 44 years. Participating pharmacists were distributed across the country with numbers according to population density (online supplemental table 1).

\section{Study population}

A total of 618 participants with T2DM were consecutively included in the study with a similar proportion of men and women $(50.7 \%$ and $49.3 \%$, respectively). Mean age in the overall population was 66.5 years, being the majority pensioners $(68.6 \%)$ and $42.4 \%$ had primary education (table 1).

Among comorbid conditions, hypertension was reported in $65.5 \%$ of participants and dyslipidemia in $53.9 \%$. The proportion of participants who reported diabetes-related comorbidities was $39.6 \%$, mainly retinal disease $(13.6 \%)$ and angina pectoris $(13.3 \%)$. Within the previous year, $14.4 \%$ of participants reported hospital admissions, $21.3 \%$ because of diabetes decompensation. In the last 2 years, $63.9 \%$ of patients had an ophthalmologic follow-up. Most of the participants (90.3\%) collected their medication within the last 3 months. Participants received a mean of 1.7 hypoglycemic antidiabetic drugs, 1.5 antihypertensive agents, and 3.4 treatments classified as "others" (table 1).

When analyzing demographic characteristics by sex, we observed that women were older, a lower proportion had tertiary studies (university), received less antihypertensive treatments (1.6 vs 1.4), and a higher proportion underwent ophthalmologic follow-ups (table 1).

\section{Adherence to antidiabetic treatments}

According to the MMAS-8, $40.6 \%$ of participants presented high adherence, $35.1 \%$ medium adherence, 
Table 1 Demographic and clinical characteristics of study participants

\begin{tabular}{|c|c|c|c|}
\hline & $\mathrm{N}=618$ & $\begin{array}{l}\text { Men } \\
\mathrm{N}=313\end{array}$ & $\begin{array}{l}\text { Women } \\
\mathrm{N}=305\end{array}$ \\
\hline \multicolumn{4}{|l|}{ Sex, n (\%) } \\
\hline Men & $313(50.7)$ & - & - \\
\hline Women & $305(49.3)$ & - & - \\
\hline Age (years), mean (SE) & $66.5(0.6)$ & $65.1(0.8)$ & $68.0(0.8)^{\star}$ \\
\hline \multicolumn{4}{|l|}{ Education level, n (\%) } \\
\hline Unschooled or not reported & $150(24.2)$ & $62(19.8)$ & $88(28.9)$ \\
\hline Primary education & $262(42.4)$ & $140(44.7)$ & $122(40.0)^{\star}$ \\
\hline Secondary education & $134(21.7)$ & $68(21.7)$ & $66(21.6)$ \\
\hline Tertiary education & $72(11.7)$ & $43(13.7)$ & $29(9.5)$ \\
\hline \multicolumn{4}{|l|}{ Employment status, n (\%) } \\
\hline Employed & $140(22.7)$ & $83(26.5)$ & $57(18.7)$ \\
\hline Unemployed & $54(8.7)$ & $27(8.6)$ & $27(8.9)$ \\
\hline Pensioner & $424(68.6)$ & $203(64.9)$ & $221(72.5)$ \\
\hline Hypertension, n (\%) & $405(65.5)$ & $210(67.1)$ & $195(63.9)$ \\
\hline Dyslipidemia, n (\%) & $333(53.9)$ & $176(56.2)$ & $157(51.5)$ \\
\hline Reported diabetes-related comorbidities, n (\%) & $245(39.6)$ & $133(42.5)$ & $112(36.7)$ \\
\hline Angina pectoris & $82(13.3)$ & $43(13.7)$ & $39(12.8)$ \\
\hline Myocardial infarction & $73(11.8)$ & $40(12.8)$ & $33(10.8)$ \\
\hline Heart failure & $69(11.2)$ & $39(12.5)$ & $30(9.8)$ \\
\hline Kidney disease & $76(12.3)$ & $41(13.1)$ & $35(11.5)$ \\
\hline Retinal disease & $84(13.6)$ & $40(12.8)$ & $844(14.4)$ \\
\hline Sensitive neuropathy & $62(10.0)$ & $31(9.9)$ & $31(10.1)$ \\
\hline Peripheral arterial disease & $64(10.4)$ & $29(9.3)$ & $35(11.5)$ \\
\hline Diabetes duration (years), mean (SE) $(\mathrm{N}=515) \dagger$ & $13.6(0.5)$ & $13.3(0.7)$ & $14.0(0.7)$ \\
\hline Hospital admission (last year), n (\%) & $89(14.4)$ & $46(14.7)$ & $43(14.1)$ \\
\hline Diabetes decompensation & $19(3.0)$ & $9(2.9)$ & $10(3.3)$ \\
\hline Ophthalmological follow-up (last 2 years), n (\%) & $395(63.9)$ & $184(58.8)$ & $211(69.1)^{\star}$ \\
\hline Recent laboratory tests (last year), n (\%) & $324(52.4)$ & $160(51.1)$ & $164(53.8)$ \\
\hline Collected the medication (last 3 months), $\mathrm{n}(\%)(\mathrm{N}=574) \dagger$ & $558(90.3)$ & $281(89.9)$ & $277(90.8)$ \\
\hline \multicolumn{4}{|l|}{ No of treatments per patient, mean (SE) } \\
\hline Antidiabetic & $1.7(0.03)$ & $1.7(0.05)$ & $1.8(0.05)$ \\
\hline Insulin & $101(16.3)$ & $39(17.0)$ & $62(16.0)$ \\
\hline Oral & $517(83.7)$ & $191(83.0)$ & $326(84.0)$ \\
\hline Antihypertensive in patients with hypertension & $1.5(0.04)$ & $1.6(0.07)$ & $1.4(0.05)^{\star}$ \\
\hline Other & $3.4(0.13)$ & $3.4(0.17)$ & $3.4(0.19)$ \\
\hline \multicolumn{4}{|l|}{ Adherence (MMAS-8)‡, n (\%) } \\
\hline Low $(<6)$ & $150(24.3)$ & $80(25.6)$ & $70(23.0)$ \\
\hline Medium (6-7) & $217(35.1)$ & $116(37.1)$ & $101(33.1)$ \\
\hline High $(\geq 8)$ & $251(40.6)$ & $117(37.4)$ & $134(43.9)$ \\
\hline \multicolumn{4}{|l|}{ Hypoglycemia awareness (Clarke test), $n(\%)(N=378) \dagger$} \\
\hline Impaired $(\geq 4)$ & 94 (24.9) & $44(23.8)$ & $50(25.9)$ \\
\hline Undetermined $(=3)$ & $82(21.7)$ & $38(20.5)$ & $44(22.8)$ \\
\hline Adequate $(\leq 2)$ & $202(53.4)$ & $103(55.7)$ & 99 (51.3) \\
\hline
\end{tabular}

The Clarke test was administered to patients treated with hypoglycemic antidiabetic treatments.

*Significant differences $(95 \% \mathrm{Cl})$ calculated with the $\chi^{2}$ test for categorical variables or the Student t-test for continuous variables.

†Number of patients with available data. For the stratified analysis by sex: number of treatments per patient (men $\mathrm{N}=289$; women $\mathrm{N}=285$ ), hypoglycemia awareness (men $\mathrm{N}=185$; women $\mathrm{N}=193$ ), diabetes duration (men $\mathrm{N}=269$; women $\mathrm{N}=246$ ).

$\ddagger 2006$ Donald E. Morisky.

MMAS-8, 8-item Morisky Medication Adherence Scale. 
and $24.3 \%$ low adherence (table 1 ). No significant differences in adherence rates were observed between men and women neither in the MMAS- $8(\mathrm{p}=0.253)$, nor in dispensed adherence $(\mathrm{p}=0.786)$.

\section{Comparative analysis of adherence: MMAS- 8 versus dispensed medications}

Among participants with low adherence by dispensed medications, $57 \%$ presented low adherence with the MMAS-8 test, $14 \%$ medium adherence, and 29\% high adherence. Participants with medium adherence by dispensed medications showed medium adherence with the MMAS- 8 in $28 \%$ of participants, low in $44 \%$, and high in $28 \%$. Among participants with high adherence by dispensed medications, $43 \%$ showed high adherence with the MMAS-8, 35\% medium adherence, and 22\% low adherence. The comparison between adherence measured with the MMAS- 8 test and dispensed medications categorized by sex is shown in online supplemental table 2.

\section{Hypoglycemia awareness}

Participants treated with hypoglycemic antidiabetic treatments (sulfonylureas, glinides, and insulin; $\mathrm{n}=378,61 \%$ ) completed the Clarke questionnaire. Among them, 53.4\% showed adequate hypoglycemia awareness ( $\mathrm{R}$ responses $\leq 2), 21.7 \%$ undetermined awareness ( $\mathrm{R}$ responses $=3$ ), and $24.9 \%$ IAH ( $\mathrm{R}$ responses $\geq 4$ ) (table 1 ). The proportion of participants with severe hypoglycemia episodes without loss of consciousness in the last 6 months was $39.1 \%$, whereas $15.6 \%$ had severe hypoglycemia episodes with loss of consciousness in the last 12 months.

\section{Healthcare counseling}

The proportion of subjects who had some healthcare counseling was $70.9 \%$; $69.0 \%$ corresponded to diabetes education, $19.9 \%$ to pharmacotherapeutic assessment, and $11.2 \%$ to physician referral (table 2).

Participants with IAH showed higher physician referrals and lower diabetes education. Those with low adherence were more frequently subjected to pharmacotherapeutic assessments and physician referrals. All the subjects with both IAH and low adherence received some professional counseling, mainly diabetes education $(50.0 \%)$ and physician referral $(41.2 \%)$. In participants with neither IAH nor low adherence, the most common intervention was diabetes education (79.2\%) (table 2).

\section{Determinants of adherence to antidiabetic treatment}

In the simple model, longer diabetes duration (OR 0.98; $95 \%$ CI 0.96 to 0.99 ), receiving other treatments (OR $0.91 ; 95 \%$ CI 0.84 to 0.99 ), adequate hypoglycemia awareness (OR 0.34; $95 \%$ CI 0.20 to 0.60 ), and being attended at urban pharmacies (OR $0.54 ; 95 \%$ CI 0.37 to 0.79 ) were significant predictors of better adherence to antidiabetic treatments. In contrast, participants unschooled or without reported education were less likely to be adherent to antidiabetic treatments (OR 1.79; 95\% CI 1.13 to 2.83) (table 3 ). In the multivariable model, only hypoglycemia awareness remained significant (OR 0.40; 95\% CI 0.20 to 0.80 ) (table 3 ). Online supplemental table 3 shows the determinants of low adherence to antidiabetic treatment stratified by participant sex.

In the simple model, the type of treatment (hypoglycemic vs non-hypoglycemic agents) did not correlate with treatment adherence (OR $0.76 ; 95 \%$ CI 0.37 to 1.54 ). However, participants receiving non-hypoglycemic antidiabetic agents with longer diabetes duration were more likely to be adherent (OR $0.92 ; 95 \%$ CI 0.87 to 0.97 ) and those with tertiary education were less likely to be adherent (OR 2.80; 95\% CI 1.16 to 6.75). In the multivariable model, participants treated with sulfonylureas, glinides, or insulin attended at urban pharmacies were more likely to be adherent to antidiabetic treatments (OR 0.23; 95\% CI 0.12 to 0.44 ) (online supplemental table 4$)$.

\section{Determinants of impaired hypoglycemia awareness}

Hypoglycemia unawareness did not depend on participants' sex (OR 1.12; $95 \%$ CI 0.70 to 1.79 ). In the simple model, being unschooled or without reported education (OR 2.37; 95\% CI 1.36 to 4.13) and reporting diabetesrelated comorbidities (OR 1.60; 95\% CI 1.00 to 2.55)

Table 2 Comparison of healthcare counseling received in the overall population and in patients with low adherence and impaired hypoglycemia awareness

\begin{tabular}{|c|c|c|c|c|c|}
\hline $\begin{array}{l}\text { Healthcare counseling, } \\
\text { n (\%) }\end{array}$ & $\begin{array}{l}\text { Total } \\
\mathrm{N}=618\end{array}$ & $\begin{array}{l}\text { Impaired } \\
\text { hypoglycemia } \\
\text { awareness } \\
(\mathrm{N}=60)\end{array}$ & $\begin{array}{l}\text { Low } \\
\text { adherence } \\
(\mathrm{N}=116)\end{array}$ & $\begin{array}{l}\text { Both* } \\
(\mathrm{N}=34)\end{array}$ & $\begin{array}{l}\text { None } \\
(\mathrm{N}=229)\end{array}$ \\
\hline None & $180(29.1)$ & $5(8.3)$ & $7(6.0)$ & 0 & $104(45.4)$ \\
\hline Healthcare counseling $\dagger$ & $438(70.9)$ & $55(91.7)$ & $109(94.0)$ & $34(100)$ & $125(54.6)$ \\
\hline Diabetes education & $302(69.0)$ & $29(52.7)$ & $47(43.1)$ & $17(50.0)$ & $99(79.2)$ \\
\hline Physician referral & 49 (11.2) & $14(25.5)$ & $15(13.7)$ & 14 (41.2) & $6(4.8)$ \\
\hline
\end{tabular}

*Number of patients with low adherence and impaired hypoglycemia awareness.

†Percentages are calculated over the total number of patients who received healthcare advice. 
Table 3 Determinants of low adherence to antidiabetic treatment obtained with simple and multivariable regression models

\begin{tabular}{|c|c|c|}
\hline Sociodemographic and clinical variables $(\mathrm{N}=618)$ & OR (95\% Cl) & \\
\hline Sex (participants) & Simple & Multivariable \\
\hline Men & Ref. & - \\
\hline Women & 0.87 (0.60 to 1.25$)$ & - \\
\hline \multicolumn{3}{|l|}{ Sex (pharmacists) } \\
\hline Men & Ref. & - \\
\hline Women & $1.03(0.70$ to 1.51$)$ & - \\
\hline \multicolumn{3}{|l|}{ Type of pharmacy } \\
\hline Rural & Ref. & Ref. \\
\hline Urban & $0.54(0.37 \text { to } 0.79)^{\star *}$ & 0.62 (0.36 to 1.05$)$ \\
\hline Diabetes duration (years), (0-92) & $0.98(0.96 \text { to } 0.99)^{*}$ & 0.99 (0.96 to 1.01$)$ \\
\hline Age (years), (18-99) & $1.00(0.98$ to 1.01$)$ & - \\
\hline \multicolumn{3}{|l|}{ Education level } \\
\hline Unschooled or not reported & $1.79(1.13 \text { to } 2.83)^{*}$ & 1.73 (0.99 to 3.03$)$ \\
\hline Primary & Ref. & Ref. \\
\hline Secondary & 1.27 (0.77 to 2.09$)$ & 1.15 (0.64 to 2.06$)$ \\
\hline Tertiary & 1.55 (0.85 to 2.83$)$ & $1.65(0.84$ to 3.26$)$ \\
\hline \multicolumn{3}{|l|}{ Employment situation } \\
\hline Pensioner & Ref. & Ref. \\
\hline Employed & $1.52(0.99$ to 2.34$)$ & 1.60 (0.94 to 2.71$)$ \\
\hline Unemployed & 1.37 (0.72 to 2.59$)$ & $1.26(0.60$ to 2.61$)$ \\
\hline Hypertension (ref. no) & $1.16(0.78$ to 1.71$)$ & - \\
\hline Dyslipidemia (ref. no) & $1.08(0.75$ to 1.50$)$ & - \\
\hline Reported diabetes-related comorbidities (ref. no) & 0.85 (0.58 to 1.24$)$ & - \\
\hline Hospital admission (last year) (ref. no) & 1.44 (0.88 to 2.36$)$ & - \\
\hline Recent laboratory analyses (last year) (ref. no) & 0.74 (0.51 to 1.07$)$ & - \\
\hline Use of hypoglycemic agents & $0.76(0.37$ to 1.54$)$ & \\
\hline \multicolumn{3}{|l|}{ No of treatments per patient } \\
\hline Hypoglycemic (0-5) & 0.86 (0.69 to 1.08$)$ & - \\
\hline Antihypertensive (0-7) & $1.10(0.89$ to 1.37$)$ & - \\
\hline Other $(0-20)$ & $0.91(0.84 \text { to } 0.99)^{*}$ & - \\
\hline \multicolumn{3}{|l|}{ Hypoglycemia awareness (Clarke test) } \\
\hline Impaired $(\geq 4)$ & Ref. & Ref. \\
\hline Undetermined $(=3)$ & $0.65(0.34$ to 1.23$)$ & 0.88 (0.38 to 1.83$)$ \\
\hline Adequate $(\leq 2)$ & $0.34(0.20 \text { to } 0.60)^{* \star *}$ & $0.40(0.20 \text { to } 0.80)^{*}$ \\
\hline Non-reported & 0.60 (0.36 to 1.00$)$ & 0.80 (0.40 to 1.62$)$ \\
\hline
\end{tabular}

Diabetes duration was not included in the multivariable model because it presented a high proportion of missing values.

Numbers in bold indicate statistical significance. $P$ value: ${ }^{*} p<0.05 ;{ }^{* *} p<0.01 ;{ }^{* * *} p<0.001$.

were significantly associated with IAH. Factors significantly associated with adequate hypoglycemia awareness were age (OR $0.99 ; 95 \%$ CI 0.97 to 0.99 ), being attended in an urban pharmacy (OR 0.54 ; $95 \%$ CI 0.24 to 0.62 ), and having high adherence to antidiabetic treatments (OR 0.24; 95\% CI 0.12 to 0.46 ) (table 4).

In the multivariable model, a lower educational level (OR 2.04; 95\% CI 1.07 to 3.86) and the presence of comorbidities (OR 1.82; 95\% CI 1.08 to 3.08) continued to be associated with an impaired recognition of hypoglycemia, whereas high adherence was a significant factor of hypoglycemia awareness (OR $0.27 ; 95 \%$ CI 0.13 to 0.54 ) (table 4). Age was not included in the model due to collinearity. Specific determinants of hypoglycemia unawareness in the men's multivariable model were being unemployed (OR 3.53; 95\% CI 1.21 to 10.30), unschooled or without reported education (OR 3.20; $95 \%$ CI 1.10 to 8.49), and reporting diabetes-related comorbidities (OR 
Table 4 Determinants of impaired hypoglycemia awareness obtained with simple and multivariable regression models OR $(95 \% \mathrm{Cl})$

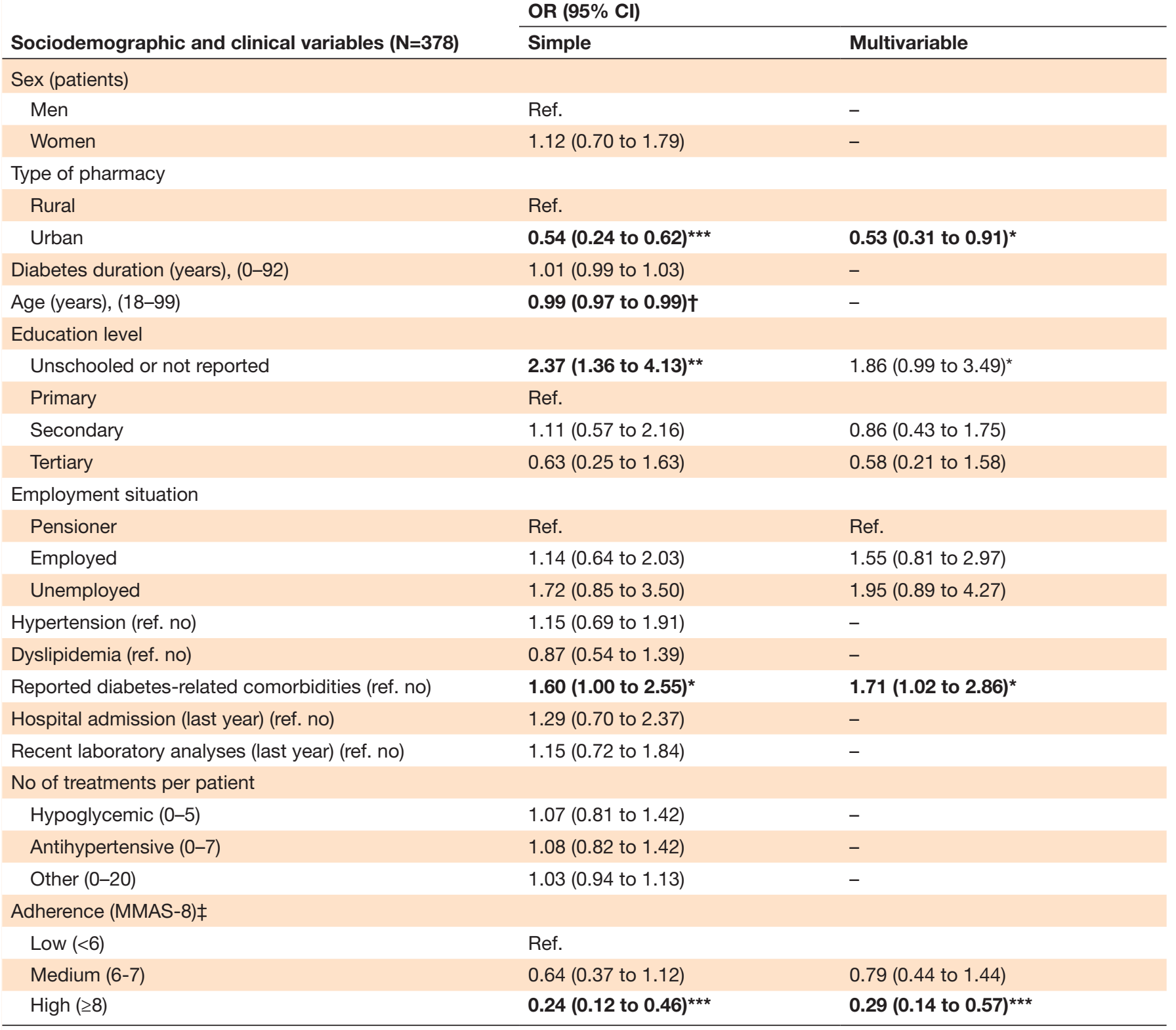

Numbers in bold indicate statistical significance. $P$ value: ${ }^{*} p<0.05 ;{ }^{* *} p<0.01 ;{ }^{* * *} p<0.001$.

$\dagger$ Not included in the multivariable analysis because of collinearity. $\ddagger 2006$ Donald E. Morisky.

MMAS, Morisky Medication Adherence Scale.

2.64; $95 \%$ CI 1.26 to 5.54$)$ in the women's multivariable model (online supplemental table 5).

\section{DISCUSSION}

This nationwide study conducted in community pharmacies of Spain assessed the level of adherence to antidiabetic treatments and of hypoglycemia awareness in people with T2DM, and their possible associated factors.

Adherence estimates vary widely between studies (43\%-86\%), depending on the target population and adherence definition. ${ }^{28}$ In a large cross-sectional study conducted in Spain, $65.4 \%$ of individuals were adherent to antidiabetic treatments ${ }^{29}$ and, in our study, the MMAS-8 showed a correct adherence in only $41 \%$ of the studied T2DM population.

Given the low adherence to antidiabetic treatments reported, assessing associated factors can be crucial to implement preventive strategies. In our models, hypoglycemia unawareness was less frequent in participants with high adherence to T2DM treatment, suggesting a correlation between both conditions. Another significant determinant of adherence found in this study is the type of pharmacy, with urban community pharmacies showing higher adherence rates. One of the factors that could explain this result relates to a higher educational level in urban populations, which agrees with the fact that 
participants treated with sulfonylureas, glinides, or insulin and attended at urban pharmacies were more likely to be adherent to antidiabetic treatments, assuming that adherence to risky or complex treatments partly depends on how informed patients are. Previous predictors of non-adherence found in other studies include male sex, higher education, higher daily total pill burden, use of mail-order versus retail pharmacies, ${ }^{31}$ younger age, and more frequent hypoglycemia. ${ }^{32}$ Among these factors, the models presented in this study only identified the education level and the number of treatments per patient as significantly correlated with adherence to antidiabetic treatments, maybe because of the different sample size and setting.

The concordance between adherence rates obtained with electronic records of dispensed medications and the MMAS-8 was low, with information from dispensed medications having poor discriminative power. These results may be explained considering that T2DM treatments are entirely covered for pensioners in the Spanish healthcare system, representing a minimal out-of-pocket contribution for the rest of people. Then, adherence data corroborate that the collection of treatments from the pharmacy does not assure their administration, as some people may be collecting the medicines from the pharmacies because they are almost completely free. The discrepancy between MMAS-8 data and electronic records of dispensed medications should prompt educational interventions in Spain and countries with similar healthcare systems to increase self-responsible pharmacological use and adherence.

The Clarke test results reported a $25 \%$ rate of hypoglycemia unawareness in people with T2DM treated with hypoglycemic agents, a similar figure to that described previously for type $1 \mathrm{DM}$ (T1DM). ${ }^{33}$ The comparison of this rate with those reported in other studies is challenged by the scarce data available on the presence of IAH in people with T2DM. Of the few examples are a recent Dutch nationwide cohort study (2350 people) using the same validated test and describing an almost $10 \%$ prevalence of IAH in people with T2DM on insulin therapy ${ }^{34}$ or the cross-sectional study performed at nine Primary Care Centers in Spain showing an IAH prevalence of $10 \%-12 \% .{ }^{35}$ Since neuroendocrine response impairment is considered to be similar in people with advanced T2DM or T1DM, ${ }^{36}$ differences in the reported hypoglycemia awareness between our study and the Dutch cohort could be due to the more advanced population or other sociocultural factors. Of particular importance in explaining these differences is the setting (community pharmacy) where the survey was administered as it can have a great influence on study outcomes. ${ }^{37}$ In this regard, the community pharmacy is a remarkably different setting compared with those in previously published studies. Overall, the higher IAH prevalence detected in community pharmacies could point out the interest in promoting research and therapeutical interventions in other settings beyond the hospital or primary care. Moreover, these high rates of IAH in advanced populations with T2DM should support the investment in hypoglycemia detection measures, such as continuous glucose monitoring. ${ }^{38}$

We found specific risk factors of impaired hypoglycemia recognition by sex, such as lower educational level, the presence of comorbidities, or being unemployed. Our group recently reported a geographical distribution of hypoglycemia in the overall Spanish population, which was conditioned by socioeconomic factors such as unemployment and literacy/education, supporting the present results and the claimed need to consider those factors to develop preventive strategies. ${ }^{39}$

In light of the low adherence and high hypoglycemia unawareness detected at community pharmacies, pharmacist-led preventive strategies are critically important. In this context, these professionals, with appropriate training, are fully qualified to provide advice and care on correct medication use, adherence, disease-related complications, behavioral and lifestyle modifications, selfmonitoring of symptoms, and treatment-related adverse events, ${ }^{40}$ given their regular interaction with patients and vast expertise on dosage regimens and treatmentrelated side effects. ${ }^{41}$ In fact, in our study, a high proportion of participants received healthcare counseling by pharmacists, particularly those subjects with IAH and low adherence. Previous studies showed the effectiveness of pharmaceutical care in improving adherence, ${ }^{42} 43$ glycemic control, ${ }^{44}{ }^{45}$ and reducing cardiovascular risk ${ }^{44}$ in people with diabetes mellitus.

The results of this study should be interpreted considering different limitations. First, given the observational nature of the study, some variables presented a considerable proportion of missing values, such as self-reported adherence. Second, the observational and cross-sectional design precludes inferring causal inference. Third, most of the variables included are subjective and can be influenced by recall errors, and some results in the subgroup analysis are challenging to interpret with available data. Fourth, patient and pharmacist selection could have biased the results toward more regular pharmacy users and pharmacists more interested in diabetes management.

Despite these limitations, this study is one of the largest conducted in Spanish community pharmacies. The cohort of patients included is representative of the Spanish population with advanced T2DM, polypharmacy, and treated at primary care ${ }^{46}$ These data are crucial to identify non-adherent patient profiles and compare data with that obtained in other clinical settings to optimize complementary interventions. Moreover, the MMAS- 8 is a reliable and valid tool to assess adherence, which has a validated Spanish version. ${ }^{47} 48$

\section{CONCLUSIONS}

This cross-sectional study confirmed that antidiabetic treatment adherence is low and identified a significant 
prevalence of IAH in a T2DM cohort. As both conditions were effectively evaluated in community pharmacies, actions to improve adherence and hypoglycemia awareness may be conducted in these facilities. Main factors associated with low adherence and IAH were described, which may be determinant for future healthcare strategies. Further prospective research is needed to confirm the effectiveness of the intervention.

\section{Author affiliations}

${ }^{1}$ Endocrinology and Nutrition Unit, Segovia Hospital Complex, Segovia, Castilla y León, Spain

${ }^{2}$ Community Pharmacy Cangas de Morrazo, Pontevedra, Spain

${ }^{3}$ Diabetes Group of the Spanish Society of Clinical, Family and Community

Pharmacy (SEFAC), Barcelona, Spain

${ }^{4}$ Community Pharmacy Fuenlabrada, Madrid, Spain

${ }^{5}$ Diabetes Group of the Spanish Society of Family and Community Pharmacy (semFYC), Barcelona, Spain

${ }^{6}$ Diabetes Group of the Spanish Society of Primary Care Physicians (SEMERGEN), Madrid, Spain

${ }^{7}$ Department of Clinical Medicine, Miguel Hernandez University of Elche - Campus of San Juan de Alicante, San Juan, Spain

${ }^{8}$ Community Pharmacy Maella, Zaragoza, Spain

${ }^{9}$ Community Pharmacy Vigo, Vigo, Spain

Acknowledgements The authors thank the community pharmacists who participated in the study. They also thank Dr Maria Rubio Valera, Dr Ignacio Aznar Lou, and Dr Joan Domènech Abella for critically reviewing the manuscript and the statistical support provided. Carla Granados from Trialance SCCL provided medical writing assistance. The MMAS-8 Scale, content, name, and trademarks are protected by US copyright and trademark laws. Permission for use of the scale and its coding is required. A license agreement is available from MMAR, LLC., Donald E. Morisky, ScD, ScM, MSPH, 294 Lindura Ct., USA (donald.morisky@moriskyscale. com).

Contributors FG-P accepts full responsibility for the work and/or the conduct of the study, had access to the data, and controlled the decision to publish. All authors have participated sufficiently in the work presented in the manuscript to take public responsibility for the content. FG-P, JAFP, and AM contributed to the conception of the work presented in the manuscript, acquisition, analysis and interpretation of data, and drafting the article. IMSB, EAM, PM-P, IMG, and NFA-R contributed to the analysis and interpretation of data and revised the article for critically important content. All authors provided their final approval of the version to be published.

Funding This project was funded by Sanofi. The authors take full responsibility for the content and conclusions presented in the manuscript and did not receive any funding for this work.

Competing interests FG-P has taken part in advisory panels for Sanofi and Novo Nordisk; has received research support from Sanofi, Novo Nordisk, Boehringer Ingelheim Pharmaceuticals, and Lilly; and has acted as a speaker for Sanofi, Novo Nordisk, Boehringer Ingelheim Pharmaceuticals, AstraZeneca Pharmaceuticals LP, Bristol-Myers Squibb Company, and Lilly and is an editorial board member of BMJ Open Diabetes Research \& Care. The remaining authors have no conflict of interest to disclose.

\section{Patient consent for publication Not applicable.}

Ethics approval This study was approved by the Clinical Research Ethics Committee of Fundació Sant Joan de Déu (PIC/106/19).

Provenance and peer review Not commissioned; externally peer reviewed.

Data availability statement Data are available on reasonable request.

Supplemental material This content has been supplied by the author(s). It has not been vetted by BMJ Publishing Group Limited (BMJ) and may not have been peer-reviewed. Any opinions or recommendations discussed are solely those of the author(s) and are not endorsed by BMJ. BMJ disclaims all liability and responsibility arising from any reliance placed on the content. Where the content includes any translated material, BMJ does not warrant the accuracy and reliability of the translations (including but not limited to local regulations, clinical guidelines, terminology, drug names and drug dosages), and is not responsible for any error and/or omissions arising from translation and adaptation or otherwise.

Open access This is an open access article distributed in accordance with the Creative Commons Attribution Non Commercial (CC BY-NC 4.0) license, which permits others to distribute, remix, adapt, build upon this work non-commercially, and license their derivative works on different terms, provided the original work is properly cited, appropriate credit is given, any changes made indicated, and the use is non-commercial. See: http://creativecommons.org/licenses/by-nc/4.0/.

ORCID ID

Fernando Gomez-Peralta http://orcid.org/0000-0003-0106-0091

\section{REFERENCES}

1 NCD Risk Factor Collaboration (NCD-RisC). Worldwide trends in diabetes since 1980: a pooled analysis of 751 population-based studies with 4.4 million participants. Lancet 2016;387:1513-30.

2 Cho NH, Shaw JE, Karuranga S, et al. IDF diabetes atlas: global estimates of diabetes prevalence for 2017 and projections for 2045. Diabetes Res Clin Pract 2018;138:271-81.

3 American Diabetes Association, Classification and Diagnosis of Diabetes. Standards of medical care in diabetes-2018. Diabetes Care 2018:41:S13-27.

4 Soriguer F, Goday A, Bosch-Comas A, et al. Prevalence of diabetes mellitus and impaired glucose regulation in Spain: the Di@bet.es Study. Diabetologia 2012;55:88-93.

5 Pérez A, Mediavilla JJ, Miñambres I, et al. Glycemic control in patients with type 2 diabetes mellitus in Spain. Rev Clin Esp 2014;214:429-36.

6 Gomez-Peralta F, Escalada San Martín FJ, Menéndez Torre E, et al. Spanish Diabetes Society (SED) recommendations for the pharmacologic treatment of hyperglycemia in type 2 diabetes: 2018 update. Endocrinol Diabetes Nutr 2018;65:611-24.

7 Juarez DT, Tan C, Davis J, et al. Factors affecting sustained medication adherence and its impact on health care utilization in patients with diabetes. J Pharm Health Serv Res 2013:4:89-94.

8 Martyn-Nemeth P, Schwarz Farabi S, Mihailescu D, et al. Fear of hypoglycemia in adults with type 1 diabetes: impact of therapeutic advances and strategies for prevention - a review. J Diabetes Complications 2016;30:167-77.

9 Cramer JA. A systematic review of adherence with medications for diabetes. Diabetes Care 2004;27:1218-24.

10 Amiel SA, Dixon T, Mann R, et al. Hypoglycaemia in type 2 diabetes. Diabet Med 2008;25:245-54.

11 Barranco RJ, Gomez-Peralta F, Abreu C, et al. Incidence and care-related costs of severe hypoglycaemia requiring emergency treatment in Andalusia (Spain): the PAUEPAD project. Diabet Med 2015;32:1520-6.

12 Menéndez Torre E, Lafita Tejedor J, Artola Menéndez S. Recommendations for the pharmacologic treatment of hyperglycemia in type 2 diabetes. Av en Diabetol 2010;26:331-8.

13 Clarke WL, Cox DJ, Gonder-Frederick LA, et al. Reduced awareness of hypoglycemia in adults with IDDM. A prospective study of hypoglycemic frequency and associated symptoms. Diabetes Care 1995:18:517-22.

14 Lin YK, Hung M, Sharma A, et al. Impaired awareness of hypoglycemia continues to be a risk factor for severe hypoglycemia despite the use of continuous glucose monitoring system in type 1 diabetes. Endocr Pract 2019;25:517-25.

15 Cryer PE. The barrier of hypoglycemia in diabetes. Diabetes 2008:57:3169-76.

16 Monnier L, Wojtusciszyn A, Colette C, et al. The contribution of glucose variability to asymptomatic hypoglycemia in persons with type 2 diabetes. Diabetes Technol Ther 2011;13:813-8.

17 SEFAC. Available: https://www.sefac.org/

18 Sediabetes. Available: https://www.sediabetes.org/

19 Semergen. Available: https://www.semergen.es

20 SemFYC. Available: https://www.semfyc.es/medicos/

21 Ruiz D, Fornos Pérez JA, Gómez-Peralta F. The 'ADHIFAC' study protocol: assessment of adherence to glucose-lowering treatment and detection of hypoglycemia in Spanish community pharmacies. Farm Comunitarios 2020;12:5-20.

22 Martínez Pérez P, Pomares Gómez F, Orozco Beltrán D. Validación de la Morisky medication adherence scale 8-ítems (MMAS-8) para El tratamiento de la diabetes mellitus tipo 2 (DM2) en España. In: Tratamiento de la diabetes: XXVIII Congreso Nacional de la Sociedad Española de diabetes, 2017: 101-2.

23 Morisky DE, Ang A, Krousel-Wood M, et al. Predictive validity of a medication adherence measure in an outpatient setting. $J$ Clin Hypertens 2008;10:348-54. 
24 Berlowitz DR, Foy CG, Kazis LE, et al. Effect of intensive bloodpressure treatment on patient-reported outcomes. N Engl J Med 2017;377:733-44.

25 Bress AP, Bellows BK, King JB, et al. Cost-Effectiveness of intensive versus standard blood-pressure control. N Engl J Med 2017;377:745-55.

26 Jansa M, Quirós C, Giménez M. Análisis psicométrico de las versiones en lengua castellana $Y$ catalana de un cuestionario de percepción de la hipoglucemia [Psychometric analysis of the Spanish and Catalan versions of a questionnaire for hypoglycemia awareness]. Med Clin 2015;144:440-4.

27 Morisky DE, Green LW, Levine DM. Concurrent and predictive validity of a self-reported measure of medication adherence. Med Care 1986;24:67-74.

28 Davies MJ, Gagliardino JJ, Gray LJ, et al. Real-world factors affecting adherence to insulin therapy in patients with type 1 or type 2 diabetes mellitus: a systematic review. Diabet Med 2013;30:512-24.

29 Labrador Barba E, Rodríguez de Miguel M, Hernández-Mijares A, et al. Medication adherence and persistence in type 2 diabetes mellitus: perspectives of patients, physicians and pharmacists on the Spanish health care system. Patient Prefer Adherence 2017:11:707-18.

30 Pathak RD, Schroeder EB, Seaquist ER, et al. Severe hypoglycemia requiring medical intervention in a large cohort of adults with diabetes receiving care in U.S. integrated health care delivery systems: 2005-2011. Diabetes Care 2016;39:363-70.

31 Kirkman MS, Rowan-Martin MT, Levin R, et al. Determinants of adherence to diabetes medications: findings from a large pharmacy claims database. Diabetes Care 2015;38:dc142098.

32 Peyrot M, Barnett AH, Meneghini LF, et al. Factors associated with injection omission/non-adherence in the global attitudes of patients and physicians in insulin therapy study. Diabetes Obes Metab 2012;14:1081-7.

33 Geddes J, Schopman JE, Zammitt NN, et al. Prevalence of impaired awareness of hypoglycaemia in adults with type 1 diabetes. Diabet Med 2008;25:501-4.

34 van Meijel LA, de Vegt F, Abbink EJ, et al. High prevalence of impaired awareness of hypoglycemia and severe hypoglycemia among people with insulin-treated type 2 diabetes: the Dutch diabetes pearl cohort. BMJ Open Diabetes Res Care 2020;8:e000935

35 Cabré C, Colungo C, Vinagre I, et al. Frequency and awareness of hypoglycemia in patients with type 2 diabetes treated with two or more insulin injections in primary care outpatient clinics. Prim Care Diabetes 2020;14:168-72.
36 Segel SA, Paramore DS, Cryer PE. Hypoglycemia-associated autonomic failure in advanced type 2 diabetes. Diabetes 2002;51:724-33.

37 Bowling A. Mode of questionnaire administration can have serious effects on data quality. J Public Health 2005;27:281-91.

38 Gomez-Peralta F, Dunn T, Landuyt K, et al. Flash glucose monitoring reduces glycemic variability and hypoglycemia: real-world data from Spain. BMJ Open Diabetes Res Care 2020;8:e001052.

39 Gomez-Peralta F, Abreu C, Benito M, et al. Geographical clustering and socioeconomic factors associated with hypoglycemic events requiring emergency assistance in Andalusia (Spain). BMJ Open Diabetes Res Care 2021;9:e001731.

40 Shane-McWhorter L, Fermo JD, Bultemeier NC, et al. National survey of pharmacist certified diabetes educators. Pharmacotherapy 2002;22:1579-93.

41 Lindenmeyer A, Hearnshaw $\mathrm{H}$, Vermeire $\mathrm{E}$, et al. Interventions to improve adherence to medication in people with type 2 diabetes mellitus: a review of the literature on the role of pharmacists. J Clin Pharm Ther 2006;31:409-19.

42 Obreli-Neto PR, Guidoni CM, de Oliveira Baldoni A, et al. Effect of a 36-month pharmaceutical care program on pharmacotherapy adherence in elderly diabetic and hypertensive patients. Int J Clin Pharm 2011;33:642-9.

43 Taylor CT, Byrd DC, Krueger K. Improving primary care in rural Alabama with a pharmacy initiative. Am J Health Syst Pharm 2003;60:1123-9.

44 Al Mazroui NR, Kamal MM, Ghabash NM, et al. Influence of pharmaceutical care on health outcomes in patients with type 2 diabetes mellitus. Br J Clin Pharmacol 2009;67:547-57.

45 Mehuys E, Van Bortel L, De Bolle L, et al. Effectiveness of a community pharmacist intervention in diabetes care: a randomized controlled trial. J Clin Pharm Ther 2011;36:602-13.

46 Pérez A, Franch J, Cases A, et al. [Relationship between the degree of glycemic control and diabetes characteristics and hyperglycemia treatment in type 2 diabetes. DIABES Study]. Med Clin 2012;138:505-11.

47 DiBonaventura M, Wintfeld N, Huang J, et al. The association between nonadherence and glycated hemoglobin among type 2 diabetes patients using basal insulin analogs. Patient Prefer Adherence 2014;8:873.

48 Martínez Péreza P, Pomares Gómeza F, Orozco Beltránb D. Quesada ricob JA y Mira Solvesb JJ. P -162 - validación De La morisky medication adherence scale 8-Ítems (Mmas-8) para El tratamiento De La diabetes mellitus tipo 2 (Dm2) En España. Endocrinología, Diabetes y Nutrición 2017;64:2530. 